\title{
Modelling of Contact Between Stiff Bodies in Automotive Transmission Systems
}

\author{
Geoffrey Virlez, Olivier Brüls, Nicolas Poulet, Emmanuel Tromme and Pierre \\ Duysinx
}

\begin{abstract}
Many transmission components contain moving parts, which can come into in contact. For example, the TORSEN differentials are mainly composed of gear pairs and thrust washers. The friction involved by contacts between these two parts is essential in the working principle of such differentials. In this chapter, two different contact models are presented and exploited for the modelling of differentials. The former uses an augmented Lagrangian technique or a penalty method and is defined between two flexible bodies or between a rigid body and a flexible structure. The second contact formulation is a continuous impact modelling based on a restitution coefficient.
\end{abstract}

\section{Introduction}

Nowadays in automotive industries the requirements to reduce fuel consumption and environmental pollution are greatly increasing. Reducing the weight of the vehicle, lowering of mechanical losses and developing new hybrid electric propulsion systems are needed in order to reach this goal. Nevertheless these new vehicle designs should not alter the security and the comfort of the passengers. For instance, electronic control systems such as ABS or ESP involve additional automotive components and therefore tend to increase the global weight, but they highly improve

G. Virlez, O. Brüls, E. Tromme and P. Duysinx

Department of Aerospace and Mechanical Engineering (LTAS), University of Liège, Chemin des chevreuils, 1, B52/3, 4000 Liège, Belgium

e-mail: geoffrey.virlez@ulg.ac.be; o.bruls@ulg.ac.be; emmanuel.tromme@ulg.ac.be; p.duysinx@ulg.ac.be

N. Poulet

JTEKT TORSEN Europe S.A., Rue du grand peuplier,11, Parc industriel de Strpy, 7110 StrpyBracquegnies, Belgium

e-mail: npoulet@torsen.be 
the vehicle handling and allow to avoid accidents in a lot of situations. The mass reduction of structural parts can also lead to a higher flexibility, which can introduce vibrations and have an impact on the driving pleasure. Moreover, the comfort in the passenger cell can be affected by the reduction of acoustic isolation due to thinner structural pannels.

In order to find a compromise between these antagonist criteria, the current trend addresses the development of reliable simulation tools to enhance automotive design processes.

Multibody simulation techniques are frequently used to model complex automotive systems. For instance, dynamic simulations of crankshaft or connecting rods have been carried out in [18] to analyse the mechanical losses; the study of deformations and stresses are available in $[7,17]$ for global multicylinder engines. The suspensions are also widely modelled using multibody tools [3]. The models are often composed of a mixed set of rigid and flexible bodies and enable to analyse the vehicle dynamic behaviour in case of maneuvers or braking (see for example [8] and [10]).

Models of transmission components is less mature because several complex physical phenomena are involved such as stick-slip, backlash between gear teeth, contact with friction, impact or hysteresis. The modelling of these nonlinear and discontinuous effects is not trivial and can lead to numerical problems during the simulations. The development of specific formulations is needed in order to manage these particular effects. The driveline devices such as clutch, gear box or differential highly interact together. They influence the driveline behaviour and also the whole vehicle performance. For example, the differential features can have a direct influence on the sizing of anti-roll bar and suspensions. Therefore, individual models of transmission components are often not sufficient and there is a need to have global drivetrain or even full vehicle models. In this way, the driveline modelling would allow the improvement of the performance not only of the transmission devices, but also of the other subsystems of the vehicle.

In automotive as in other fields of mechanics, many transmission components include contacts between different parts. These contacts inhibit the relative motion in one or several directions but they let free the motion in the other directions. The contact can be: bilateral or unilateral, rigid or flexible, frictional or frictionless. Several complex physical phenomena can be involved by contacts. For instance, if the relative velocity when the contact occurs is high for unilateral contact, the impact encountered can generate vibration waves in the body structure. Permanent plastic deformations can be induced [20]. The friction can also lead to stick-slip phenomena due to the difference between static and dynamic friction coefficients.

These accurate and efficient contact models are essential in order to get reliable drivetrain models. Gear boxes or differentials include numerous contacts which play a key role in the working principle of these mechanisms. It can not be expected to set up a realistic dynamic model of this kind of transmission components without a good and reliable mathematical formulation of contacts.

In the literature, three main categories of contact modelling can be distinguished according to the behaviour considered to model the bodies subjected to contact: 
rigid-rigid contact [20], flexible-rigid contact [14] or flexible-flexible contact [23]. In the field of multibody systems dynamics, two different approaches are often used to formulate the contact condition: continuous contact modelling and instantaneous contact modelling. The continuous method does not need specific algorithmic tools to manage the impact phenomena. The contact forces are added in the equations of motion of the mechanism and a standard time integration scheme can be used to solve the complete system. The positions and velocities of all bodies vary continuously and it is not necessary to stop the time integration at the moment of contact establishment (see [15] for more details). With instantaneous contact models, the motion is divided into two periods, before and after the impact. While the displacements are continuous, a jump of the relative velocity is observed at the contact instant. Instantaneous contact formulations are often related to nonsmooth dynamic methods $[1,13]$. The discontinuities in the velocity field require the use of special integration methods $[12,16,5]$. For instance, event-driven approaches require the interruption of the time integration at each impact whereas time-stepping methods discretize in time the complete multibody system dynamics including the unilateral constraints and the impact forces.

The objective of this chapter is to investigate two different unilateral contact formulations for modelling contacts in dynamic simulation of automotive drivetrains. For large models including numerous contacts, it can be very useful to use various contact conditions according to the detail level needed for each contact. For instance in case of a complete drivetrain model, accurate and fine contact formulations can be used inside the components modelled in detail whereas rougher contact conditions are sufficient for the transmission devices globally represented. This combination of contact formulations enables to obtain low CPU time consuming models for global applications. In order to be used with a classical integration scheme, two continuous contact formulations have been considered in this work. The first one is an accurate contact model defined between two flexible bodies or between a rigid body and a flexible body. This contact formulation uses an augmented Lagrangian approach or a penalty method. Each flexible body is represented by a finite element mesh, that notably enables to analyse the stresses on the contact surfaces. The second contact formulation is a simpler model defined between two rigid bodies. This method is based on the continuous impact theory and uses a restitution coefficient. The friction has been taken into account in both contact elements.

The application under study to validate the two contact elements is the TORSEN differential. This kind of limited slip differential is mainly composed of gear pairs and thrust washers. The axial force produced by the helical mesh leads to contact between the lateral circular faces of toothed wheels and the various thrust washers. The friction generated between these two bodies is at the source of the locking effects, specific to the operation of TORSEN differentials. A unilateral frictional contact model is then essential to model accurately and reliably these differentials.

In the sequel of this chapter, the nonlinear finite element approach for flexible multibody systems available in SAMCEF/MECANO [9] is briefly presented in Sect. 2. The two contact formulations are respectively presented in Sect. 3 for the rigid/flexible coupled interation method and Sect. 4 for the continuous impact mod- 
elling. The working principle of TORSEN differential will be described in Sect. 5 and numerical results provided by the simulation of this transmission device are shown in Sect. 6 for both contact models.

\section{Finite Element Method in Multibody System Dynamics}

In this work, the chosen approach is based on the nonlinear finite element method for flexible multibody systems developed by Géradin and Cardona [9]. This method allows the modelling of complex mechanical systems composed of rigid and flexible bodies, kinematics joints and force elements. The degrees of freedom are the absolute nodal coordinates with respect to a unique inertial frame. Hence, there is no distinction between rigid and elastic coordinates which allows accounting in a natural way for many nonlinear flexible effects and large deformations. The cartesian rotation vector combined with an updated Lagrangian approach is used for the parametrization of rotations. This choice enables an exact representation of large rotations.

The dynamics of a system including holonomic bilateral constraints is described by Eqs. (1) and (2). The modelling of unilateral contact conditions within this formulation is addressed in the next two sections.

$$
\begin{aligned}
\mathbf{M}(\mathbf{q}) \ddot{\mathbf{q}}+\mathbf{g}^{\text {gyr }}(\mathbf{q}, \dot{\mathbf{q}})+\mathbf{g}^{i n t}(\mathbf{q}, \dot{\mathbf{q}})+\Phi_{\mathbf{q}}^{T}(p \Phi+k \lambda) & =\mathbf{g}^{\text {ext }}(t) \\
k \Phi(\mathbf{q}, t) & =\mathbf{0}
\end{aligned}
$$

where $\mathbf{q}, \dot{\mathbf{q}}$ and $\ddot{\mathbf{q}}$ are the generalized displacements, velocities and acceleration coordinates, $\mathbf{M}(\mathbf{q})$ is the mass matrix, $\mathbf{g}^{\text {gyr }}$ is the vector of gyroscopic and complementary inertia forces, $\mathbf{g}^{\text {int }}(\mathbf{q}, \dot{\mathbf{q}})$ is the vector of the internal forces, e.g. elastic and dissipations forces and $\mathbf{g}^{\text {ext }}(t)$ is the vector of the external forces. According to the augmented Lagrangian method, the constraint forces are formulated by $\Phi_{\mathbf{q}}^{T}(p \Phi+k \lambda)$ where $\lambda$ is the vector of Lagrange multipliers related to algebraic constraints $\Phi=0$; $k$ and $p$ are respectively a scaling and a penalty factor to improve the numerical conditioning.

Equations (1) and (2) form a system of nonlinear differential-algebraic equations. The solution is evaluated step by step using a second order accurate time integration scheme. For this study, the Chung-Hulbert scheme, which belongs to the family of the generalized $\alpha$-method, has been used (see [6], [2]). At each time step, a system of nonlinear algebraic equations has to be solved using a Newton-Raphson method. 


\section{Coupled Iterations Method for Node to Surface Contact Element}

For multibody simulation techniques based on a finite element approach, three geometrical configurations can be used to define the contact elements: node to node, node to surface and surface to surface contact elements. The last configuration being often related to mortar algorithms $[19,11]$.

In this section, a node to surface contact element, based on the coupled iteration method, is presented. In contrast to uncoupled iterations, the contact problem is solved at the same time as the other nonlinearities and no distinction is done between the degrees of freedom linked by contact and the other ones. An augmented Lagrangian approach is used to define the kinematic constraints related to each contact condition. The resulting system of equations is solved simultaneously for the displacements and Lagrange multipliers.

This formulation is suitable for implicit nonlinear analysis and is able to model contacts between a rigid structure and a flexible part (flexible/rigid contact) or between two flexible parts (flexible/flexible contact). Contact elements are created between the nodes on the contact surface of the first support and a flexible facet of a finite element (in case of flexible/flexible contact) or a rigid master surface (in case of flexible/rigid contact) on the second support. Bilateral but also unilateral contacts can be represented. In this last case, the kinematic constraints are active in case of effective contact and inactive when the two bodies are separated. Once the set of active constraints has been determined, the equations of motion have the structure of Eqs. (1) and (2). The contact algorithm can be decomposed in two steps.

The first step is a geometrical step, which consists in searching the projection of each slave node on the master surface (Fig. 1), computing the normal distance $\left(d_{n}\right)$ between the node and the surface, and measuring the displacement variations $\left(\Delta u_{1}, \Delta u_{2}\right)$ in the tangent directions during the current time step.

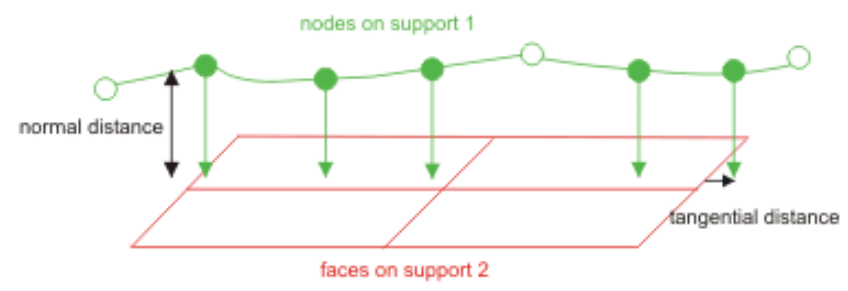

Fig. 1 Contact condition - projection of slave node on master surface

The virtual variations $\delta d_{n}, \delta \Delta u_{1}, \delta \Delta u_{2}$ can be expressed as functions of the variations of nodal unknowns $\mathbf{q}$, which are the displacements of the slave nodes, and displacements and rotations of the node linked to the rigid surface (flexible-rigid contact) or the displacements of the nodes of the facet (flexible-flexible contact). 


$$
\begin{aligned}
\delta d_{n} & =\mathbf{n}^{T} \mathbf{B} \delta \mathbf{q} \\
\delta \Delta u_{1} & =\mathbf{t}_{1}^{T} \mathbf{B} \delta \mathbf{q} \\
\delta \Delta u_{2} & =\mathbf{t}_{2}^{T} \mathbf{B} \delta \mathbf{q}
\end{aligned}
$$

where $\mathbf{n}$ is the normal, $\mathbf{t}_{1}, \mathbf{t}_{2}$ are the tangents to the surface, $\mathbf{B}=\partial\left(\mathbf{x}_{S}-\mathbf{x}_{M}\right) / \partial \mathbf{q}$, $\mathbf{x}_{S}$ and $\mathbf{x}_{M}$ being respectively the slave and master node positions expressed in the absolute reference frame.

The second step sets the contact conditions whose expression depends on the current status of the contact. For unilateral contacts, the case of an active or an inactive contact can be distinguished in the normal direction. The friction coefficient and the normal force enable to determine if the status is stick or slip in tangential directions.

In order to assess the contact status, two simple tests based on the quantities $\sigma_{n}$ and $\sigma_{t}$ are carried out. These quantities are defined as

$$
\begin{aligned}
\sigma_{n} & =k \lambda_{n}+p d_{n} \\
\sigma_{t_{1}} & =k \lambda_{t_{1}}+p \Delta u_{1} \\
\sigma_{t_{2}} & =k \lambda_{t_{2}}+p \Delta u_{2} \\
\sigma_{t} & =\sqrt{\sigma_{t_{1}}^{2}+\sigma_{t_{2}}^{2}}
\end{aligned}
$$

where $\lambda_{i}$ are three Lagrange multipliers: one for the contact $\left(\lambda_{n}\right)$ and two for the friction $\left(\lambda_{t_{1}}, \lambda_{t_{2}}\right) . k$ is a scaling factor and $p$ is a regularization parameter.

For the behaviour in the normal direction, the bold line in Fig. 2 represents the solution of the contact condition at convergence of the differential-algebraic system of equation (Eqs. 1 and 2): either the normal distance $d_{n}$ being zero or the Lagrange multiplier $\lambda_{n}$ being zero. During the iterations of the Newton-Raphson procedure, the system is not at the equilibrium and the contact criterion $\sigma_{n}$ allows to determine if the contact condition related to an active (see Sect. 3.1) or an inactive (Sect. 3.2) contact has to be used. The dotted line $\sigma_{n}=0$ divides the space in Fig. 2 in two zones. If $\sigma_{n}<0$, the node is not considered in contact and if $\sigma_{n}>0$, the node is considered in contact. The convergence property of this algorithm depends on the slope $(-p / k)$ of the line $\sigma_{n}=0$. The scaling factor $k$ being constant, the choice of the regularization parameter $p$ influences the convergence in some particular situations but the solution at convergence will not depend on this regularization parameter (cf. bold line in Fig. 2).

\subsection{Inactive Contact}

If the contact criterion $\sigma_{n}$ is positive, there is no contact nor friction forces applied on the nodes. The three Lagrange multipliers are set to zero. At the element level, the internal forces are computed by: 


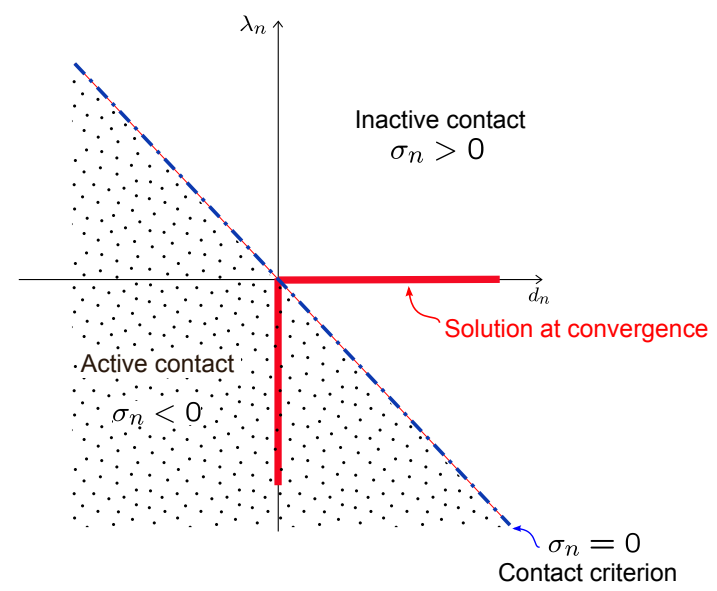

Fig. 2 Contact criterion and solution

$$
\delta \mathbf{q}^{* T} \mathbf{F}_{\text {int }}=-\left(\delta \lambda_{n} k \lambda_{n}+\delta \lambda_{t_{1}} k \lambda_{t_{1}}+\delta \lambda_{t_{2}} k \lambda_{t_{2}}\right)
$$

with:

$$
\mathbf{q}^{*}=\left\{\begin{array}{l}
\mathbf{q} \\
\lambda
\end{array}\right\}
$$

\subsection{Active Contact}

When $\sigma_{n}$ is negative, the contact is active and the kinematic constraint can be expressed by $\phi=d_{n}$. The virtual work principle enables to calculate the internal forces at the element level:

$$
\delta \mathbf{q}^{* T} \mathbf{F}_{i n t}=\delta d_{n}\left(p d_{n}+k \lambda_{n}\right)+\delta \lambda_{n} k d_{n}
$$

At the equilibrium, the kinematic constraint is satisfied $(\phi=0)$ and therefore the normal distance $d_{n}$ is equal to zero. The Lagrange multiplier $\lambda_{n}$ can be interpreted as the contact force divided by the scaling factor $k$. This last parameter is often chosen equal to the stiffness of the structural elements in order to have the same order of magnitude in the various terms of the iteration matrix of the Newton-Raphson process.

When the contact is active, two status are available for the friction behaviour: either the node is sticking to the surface or the node is sliding. The friction criterion $\sigma_{t}$ defined in Eq. (9) is used to determine the friction status. 


\subsection{Sticking Friction}

If $\sigma_{t}$ is smaller than the normal force $\sigma_{n}$ multiplied by the friction coefficient $\mu$, the node is sticking on the master face. The friction coefficient is a constant value, no distinction between the static and kinetic friction coefficient is made. Two kinematic constraints equal to the variations of tangential displacements $\left(\phi_{1}=\Delta u_{1}\right.$ and $\left.\phi_{2}=\Delta u_{2}\right)$ are introduced. As for the contact in the normal direction, the kinematic constraints are equal to zero after convergence $\left(\phi_{1}=\Delta u_{1}=0, \phi_{2}=\Delta u_{2}=0\right)$. The internal forces related to friction forces for each contact element in a sticking situation are computed from the vitual work.

$$
\begin{aligned}
\delta \mathbf{q}^{* T} \mathbf{F}_{\text {int }}= & \delta \Delta u_{1}\left(p \Delta u_{1}+k \lambda_{t_{1}}\right)+\delta \Delta u_{2}\left(p \Delta u_{2}+k \lambda_{t_{2}}\right) \\
& +\delta \lambda_{t_{1}} k \Delta u_{1}+\delta \lambda_{t_{2}} k \Delta u_{2}
\end{aligned}
$$

\subsection{Sliding Friction}

When $\sigma_{t}>\mu\left|\sigma_{n}\right|$, the slave node is sliding on the master surface. The element forces are computed from:

$$
\delta \mathbf{q}^{* T} \mathbf{F}_{i n t}=\left\{\delta \Delta u_{1} \delta \Delta u_{2} \delta \lambda_{t_{1}} \delta \lambda_{t_{2}}\right\}\left\{\begin{array}{c}
\mu\left|\sigma_{n}\right| v_{1} \\
\mu\left|\sigma_{n}\right| v_{2} \\
\left(\mu\left|\sigma_{n}\right| v_{1}-k \lambda_{t_{1}}\right) k / p \\
\left(\mu\left|\sigma_{n}\right| v_{2}-k \lambda_{t_{2}}\right) k / p
\end{array}\right\}
$$

In this case, the friction Lagrange multipliers are parallel to the variation of sliding displacements $\left(\Delta u_{1}, \Delta u_{2}\right)$. The iteration matrix for a sliding friction is not symmetrical and it is recommended to use a non-symmetric solver.

\subsection{Penalty Method}

The augmented Lagrangian approach presented above is sometimes not able to find a solution or can encounter great difficulties to converge in case of large discontinuities during the simulation. For instance, if the relative normal velocity of the colliding bodies is high at the contact establishment, an impact phenomenon occurs. This contact formulation can not manage the strong discontinuity and the rebonds after the first impact have often erratic magnitudes and frequencies. Likewise, the switching between the stick and the slip is highly nonlinear for high speed systems.

In order to improve the convergence of the algorithm, the augmented Lagrangian method can be replaced by a pure penalty method. In contrast to the Lagrangian approach where the contact is infinitely rigid, the penalty allows a small penetration between the two bodies that slightly relaxes the discontinuity. The penalty function 
can be linear or nonlinear and can be seen physically as a finite stiffness that is active in compression but not in traction. To have a smoother response, it can also be useful to account for damping in the contact model. The first step consisting of the projection of the slave nodes on the master faces is unchanged compared with Lagrangian approach.

A regularization is often used to avoid the discontinuity when the sign of the relative sliding velocity shifts (see Fig. 3). The regularized friction coefficient $\mu_{R}$ can be defined in several ways with sometimes complex functions. In this study, a simple quadratic function is used:

$$
\mu_{R}(\dot{\xi})= \begin{cases}\mu\left(2-\frac{|\dot{\xi}|}{\varepsilon_{v}}\right) \frac{\dot{\xi}}{\varepsilon_{v}} & |\dot{\xi}|<\varepsilon_{v} \\ \mu \frac{\dot{\xi}}{|\dot{\xi}|} & |\dot{\xi}| \geq \varepsilon_{v}\end{cases}
$$

where $\dot{\xi}$ is the relative sliding velocity, $\mu$ is the friction coefficient and $\varepsilon_{v}$ is the regularization tolerance.

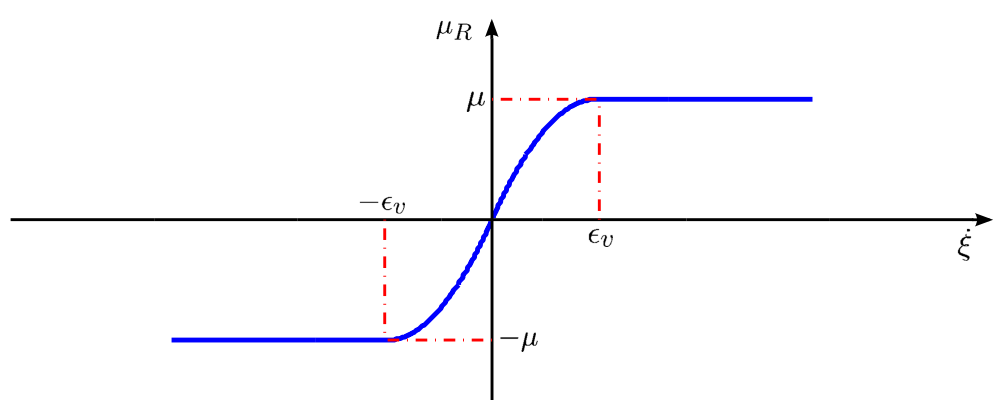

Fig. 3 Regularized friction coefficient

\section{Continuous Impact Modelling}

The continuous contact modelling is based on a continuous contact law that uses a restitution coefficient. During impacts between rigid bodies, some kinetic energy is lost. Indeed, impacts can initiate wave propagation in the bodies which absorb parts of the kinetic energy until they vanish owing to material damping. High stresses might also occur near the impact point and involve plastic deformation, which also contributes to kinetic energy loss, as well as visco-elastic material behaviour. Macro-mechanically, these various sources of kinetic energy loss are often 
summarized and expressed by a coefficient of restitution. The loss of kinetic energy described by the coefficient of restitution depends on the shapes and material properties of the colliding bodies as well as on their relative velocities. However, the restitution coefficient cannot be computed within the multibody system simulation. It has to be roughly estimated from experience, measured by experiments or determined by numerical simulations on a fast time scale [20].

There exist different definitions for the coefficient of restitution: kinematic $\left(e_{N}\right)$, kinetic $\left(e_{P}\right)$ or energetic $\left(e_{E}\right)$ :

$$
\begin{aligned}
& e_{N}=-\frac{\dot{g}_{n_{e}}}{\dot{g}_{n_{s}}} \\
& e_{P}=\frac{\Delta P_{r}}{\Delta P_{c}}=\frac{\int_{c_{c}}^{t_{e}} F \mathrm{~d} t}{\int_{t_{s}}^{t_{c}} F \mathrm{~d} t} \\
& e_{E}^{2}=-\frac{T_{r}}{T_{c}}=-\frac{\int_{t_{c}}^{t_{e}} F \dot{g}_{n} \mathrm{~d} t}{\int_{t_{s}}^{t_{c}} F \dot{g}_{n} \mathrm{~d} t}=-\frac{\int_{h_{c}}^{h_{e}} F \mathrm{~d} t}{\int_{h_{s}}^{h_{c}} F \mathrm{~d} t}
\end{aligned}
$$

where $\dot{g}_{n_{s}}$ and $\dot{g}_{n_{e}}$ are respectively the relative velocity between the two bodies in normal direction before and after impacts; the time intervals $\left[t_{s}, t_{c}\right]$ and $\left[t_{c}, t_{e}\right]$ correspond to the compression and restitution phases; $\Delta P_{c}$ and $\Delta P_{r}$ are the impulse during the compression and restitution phases; $T_{c}$ and $T_{r}$ are the deformation energies during the compression and restitution phases; $F$ is the contact force and $h=-g_{n}$ is the penetration allowed between the two bodies.

An impact with $e=1$ means no energy loss (complete elastic contact), whereas $e=0$ corresponds to a total loss of energy (plastic or inelastic contact), $0 \leq e \leq 1$.

These three forms of the restitution coefficient are equivalent unless the configuration is eccentric and the direction of slip varies during impact or if the bodies are rough. Some differences can also appear in case of frictional contact or if several impacts occur simultaneously (see [21] for more details).

\subsection{Force Law}

A penalty approach is used for this continuous contact model whereby a small penetration $h$ is allowed. The contact force is computed from this local penetration by a force law.

$$
F(h, \dot{h})=k h^{n}+c h^{n} \dot{h}
$$

where $k$ is the contact stiffness and $c$ is a damping parameter.

In order to avoid a jump at the beginning of the impact and tension force at the end of the impact, the classical viscous damping term $(c \dot{h})$ has been multiplied by $h^{n}$. 
As depicted in Fig. 4, this force law yields a hysteresis loop with $h_{s}=h_{e}=0$ for the force-penetration curve. The enclosed area represents the kinetic energy loss during impact.

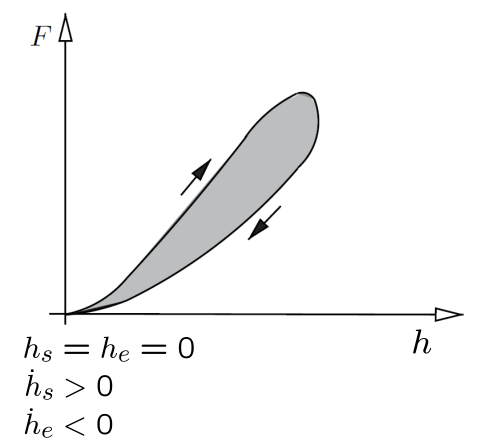

Fig. 4 Force law for continuous impact modelling

The parameters $k$ and $c$ have to be chosen in order to have realistic values for the impact duration, the local penetration and the kinetic energy loss. One way to set the damping parameter consists in formulating this coefficient as a function of the restitution coefficient. According to the contact configuration, various expressions are available in the literature (see for example [15]). For the contact considered in this work between gear wheels and washers, the expression (20) seems relevant and yields to a good approximation of the kinetic energy loss for large kinematic restitution coefficients $(e>0.8)$.

$$
c=\frac{3\left(1-e^{2}\right)}{4} \frac{k}{\dot{h}_{s}}
$$

where $\dot{h}_{s}$ is the relative normal velocity between bodies at the contact beginning. The force (Eq. 19) applied on the two bodies while there are in contact as well as the contribution of this contact element to the global iteration matrix of the system have to be specified in the contact subroutine. In order to compute the tangent stiffness matrix and the damping matrix included in the iteration matrix of the contact element, the incremental form of the virtual work principle can be used.

$$
\begin{aligned}
& \delta \mathrm{d} W=\delta \mathrm{d} h F(h, \dot{h})+\delta h \mathrm{~d} F(h, \dot{h}) \\
& \delta \mathrm{d} W=\delta \mathrm{d} h F(h, \dot{h})+\delta h\left(\frac{\partial F}{\partial h} \mathrm{~d} h+\frac{\partial F}{\partial \dot{h}} \mathrm{~d} \dot{h}\right)
\end{aligned}
$$

This last expression has to be handled in order to obtain Eq. (23) and to identify the tangent stiffness matrix and damping matrix. 


$$
\delta \mathrm{d} W=\delta \mathbf{q}^{T} \underbrace{\frac{\partial \mathbf{F}_{i n t}}{\partial \mathbf{q}}}_{\mathbf{K}_{T}} \mathrm{~d} \mathbf{q}+\delta \mathbf{q}^{T} \underbrace{\frac{\partial \mathbf{F}_{i n t}}{\partial \dot{\mathbf{q}}}}_{\mathbf{C}_{T}} \mathrm{~d} \dot{\mathbf{q}}
$$

Here $\mathbf{q}$ is the vector of nodal degree of freedom used by the contact element. In our current implementation, this vector contains the position parameters of the node located at the center of the contact surface of two bodies candidate to con-

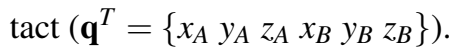

In summary, the tangent stiffness and damping matrices can be expressed in the following form:

$$
\begin{aligned}
\delta \mathrm{d} W= & \delta \mathbf{q}^{T} \underbrace{\left[\left(-\frac{F}{h}+\frac{\partial F}{\partial h}-\frac{\partial h}{\partial \dot{h}} \frac{\dot{h}}{h}\right) \mathbf{n} \mathbf{n}^{T}+\frac{F}{h} \mathbf{I}+\frac{\partial F}{\partial \dot{h}} \mathbf{n} \frac{\dot{\mathbf{x}}_{A B}^{T}}{h} \mathbf{I}\right]}_{\mathbf{K}_{T}} \mathrm{~d} \mathbf{q} \\
& +\delta \mathbf{q}^{T} \underbrace{\left[\frac{\partial F}{\partial \dot{h}} \mathbf{n}^{T}\right]}_{\mathbf{C}_{T}} \mathrm{~d} \dot{\mathbf{q}}
\end{aligned}
$$

with $\mathbf{x}_{A B}^{T}=\left\{\begin{array}{lll}x_{B}-x_{A} & y_{B}-y_{A} \quad z_{B}-z_{A}\end{array}\right\}$, the vector between nodes $A$ and $B ; \mathbf{n}$ is the normal direction to the contact surface.

\subsection{Friction Force}

The friction force produced by the contact between the two rigid bodies can be easily added to the contact element presented in the previous section. Its magnitude is given by Eq. (25) where $F_{\text {norm }}$ is equivalent to the contact force ( Eq. (19) ).

$$
F_{f r}=\mu_{R}\left|F_{\text {norm }}\right|
$$

This friction force is applied on a point $M$ located at the middle of the segment $A B$ and its direction is aligned with the tangential velocity vector $\mathbf{v}_{t}$.

\section{Description of TORSEN Differentials}

The two essential functions of a differential are to transmit the motor torque to the two output shafts and to allow a difference of rotation speed between these two outputs. In a vehicle, this mechanical device is particularly useful in turn when the outer wheels have to rotate quicker than the inner wheels to ensure a good handling.

The main drawback of a conventional differential (open differential) is that the total amount of available torque is always split between the two output shafts with 
the same constant ratio. In particular, this is a source of problems when the driving wheels have various conditions of adherence. If the motor torque exceeds the maximum transferable torque limited by road friction on one driving wheel, this wheel starts spinning. Although they do not reach their limit of friction, the other driving wheels are not able to transfer more torque because the input torque is often equally split between the two output shafts.

The TORSEN differentials significantly reduce this undesirable side effect. This kind of limited slip differential allows a variable distribution of motor torque depending on the available friction of each driving wheel. For a vehicle with asymmetric road friction between the left and right wheels, for example, right wheels are on a slippery surface (snow, mud...) whereas left wheels have good grip conditions, it is possible to transfer an extra torque to the left lane. That allows the vehicle to move forward whereas it would be hardly possible with an open differential. However, the overall driving torque can not be applied on one output shaft while no load is exerted on the second shaft. When the difference between the 2 output torques becomes too large, the differential unlocks and lets different rotation speeds but keeps the same constant torque ratio.

When a TORSEN differential is used, the torque biasing is always a precondition before any difference of rotation speed between the two output shafts. Contrary to viscous coupling, TORSEN (a contraction of Torque-Sensing) is an instantaneous and pro-active process which acts before wheel slip.

The differential can be used either to divide the drive torque into equal parts acting on the traction wheels of the same axle, or to divide the output torque from the gearbox between the two axles of four-wheels drive vehicles. This second application is often called the transfer box differential or central differential.

As depicted in Fig. 5, the TORSEN differential contains a housing in two parts as well as several gear pairs and thrust washers. Due to the axial force produced by the helical mesh, several gear wheels can move axially and enter in contact with the various thrust washers fixed on the case or housing. The friction encountered by this relative sliding is at the origin of the locking effect of TORSEN differentials. The second important contribution to the limited slip behaviour is due to the friction between the planet gears and the housing holes in which they are inserted. When one axle tries to speed up, all encountered frictions tend to slow down the relative rotation and involve a variable torque distribution between the output shafts. The biasing on the torque only results from the differential gearing mechanical friction.

This limited slip differential has four working modes which depend on the direction of torque biasing and on the drive or coast situation. According to the considered mode, the gear wheels rub against one or the other thrust washers which can have different friction coefficients and contact surfaces. 


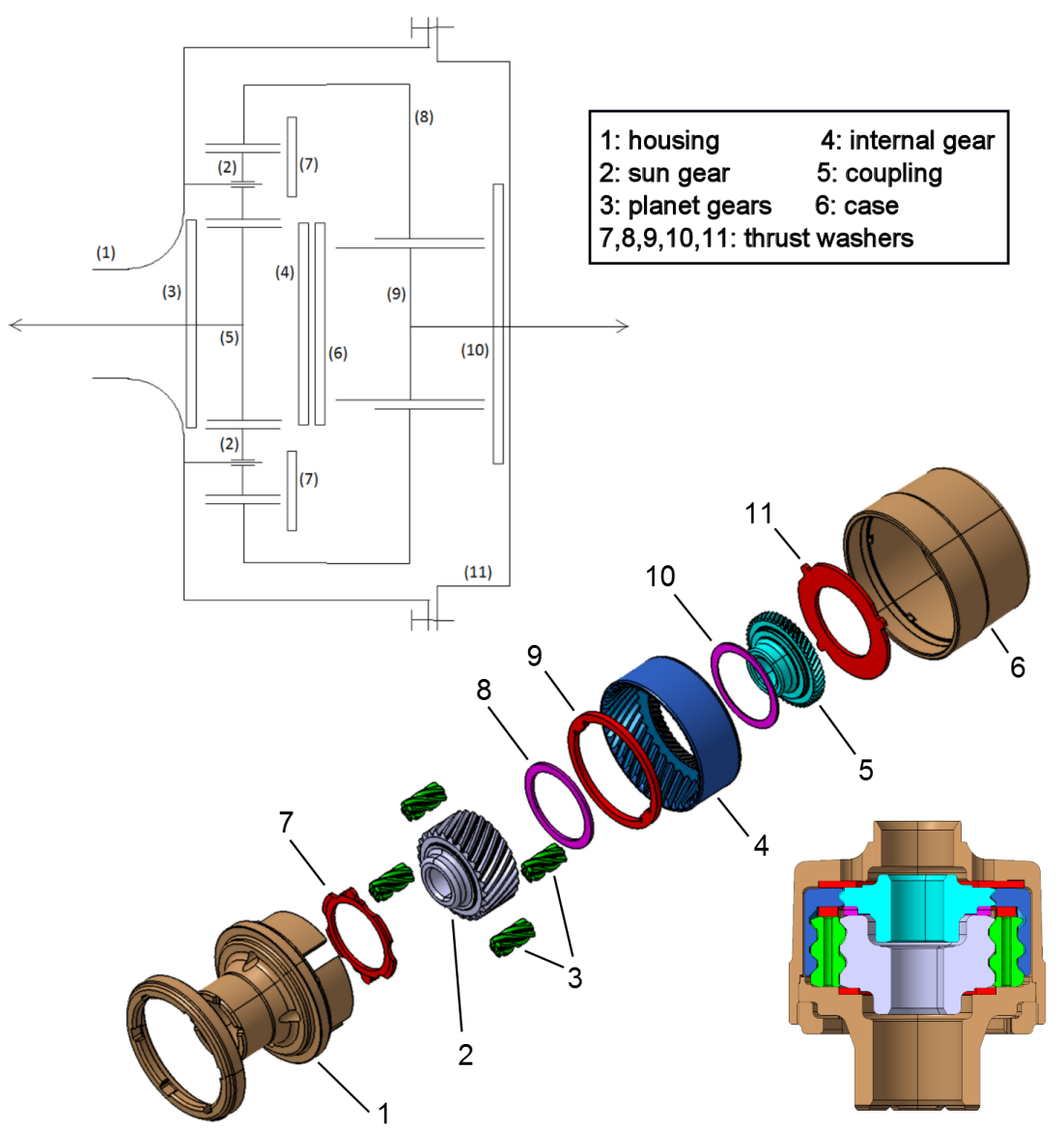

Fig. 5 Kinematic diagram, exploded view and cut-away view of type C TORSEN differential

\section{Numerical Results}

The two main kinematic constraints needed to model TORSEN differentials are gear pairs and contact conditions. The formulation used to model each gear pair is available for describing flexible gear pairs in 3 dimensional analysis of flexible mechanism. This gear element is developed in [4] and is a global kinematic joint defined between two physical nodes: one at the center of each gear wheel which is represented as a rigid body. Nevertheless the flexibility of the gear mesh is accounted for by a nonlinear spring and damper element inserted along the instantaneous normal pressure line. Several specific phenomena in gear pairs which influence significantly the dynamic response of gears are also included in the model: backlash, mesh stiffness fluctuation, misalignment, friction between teeth. Contacts between the thrust 
washers and the circular lateral faces of gear wheels have been successively modelled by the two contact formulation presented in this chapter.

A dynamic analysis of the type C TORSEN has been carry out when this component is considered in the vehicle configuration. The other driveline devices such as driveshafts are not represented in this work. Equivalent loads are applied on the differential inputs and outputs: a torque is applied on the housing whereas the rotation speed of sun gear and coupling are prescribed. The housing torque is equivalent to the driving force coming out from the gear box and transmitted to the differential housing through the propeller shaft. The prescribed rotation speeds can be seen as a measure of the adherence properties on the front and rear axles.

The time evolution of these loads has been chosen in order to study the four working modes of the differential during the same simulation and the transient behaviour at the switching time between two modes. Besides, in order to test the robustness of the model, the torque on the housing is applied with fast increasing or decreasing phases interrupted by steady state periods (see Fig. 6).
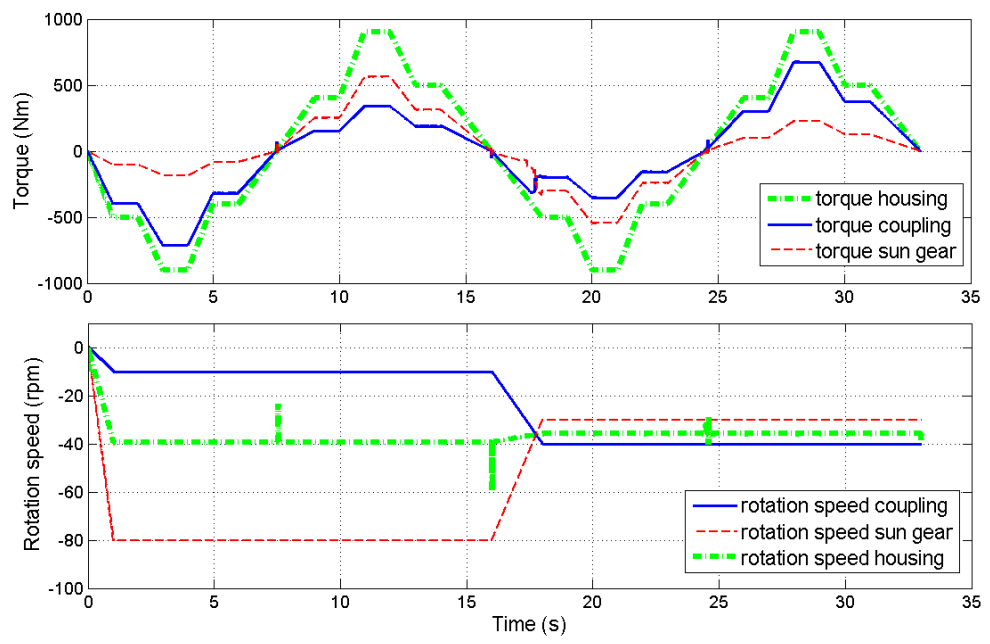

Fig. 6 Time evolution of torque and rotation speed of housing and output shafts of type C TORSEN on vehicle configuration

Some numerical results specific to the contact formulations studied in this chapter are presented in Sect. 6.1 for the coupled iterations method and in Sect. 6.2 for the continuous impact modelling.

A previous work addresses the development of full type B and type C TORSEN differential models. See Reference [22] for more details on the construction of theses models and their global validation by comparison with experimental data. 


\subsection{Flexible-Rigid Contact}

In the differential model, the five thrust washers are modelled with finite elements whereas the gear wheels are represented by rigid bodies. Therefore, the flexiblerigid version of the coupled iterations method for node to surface contact (Sect. 3) is used.

Owing to the high axial velocity of the gear wheels when the differential switches from one working mode to another one, the augmented Lagrangian approach has to be replaced by a full penalty method in order to allow the convergence of the integration algorithm as explained in Sect. 3.5. A damping force has also been used, besides the linear penalty function. The penalty function enable small interferences between the gear wheel and the thrust washer whereas the damping tends to slow down the impact velocity. The chosen damping function is a linear function with respect to the penetration velocity. The damping force has even been introduced before the effective contact to anticipate the latter and reduce the shock phenomenon. This anticipating damping has been used to facilitate the convergence but has also a physical meaning. Indeed, in real operation the film of lubricating oil between the contacting surfaces tends to slow down the bodies before the contact and then plays the role of a damper. The damping coefficient used in this model should be identified with the oil properties to have a realistic damping behaviour. Nevertheless, in this work the damping coefficient has been chosen in order to allow the convergence but without a reference with the physical properties. The contact stiffness value used to enable the small penetration has also been set only to ensure the convergence. If this value is too large, the discontinuity is not sufficiently relaxed and if the value is too small the penetration of the two contacting bodies is too high which is in contradiction with the prescribed contact condition. A regularization of the friction coefficient has been needed to avoid a large discontinuity when the relative rotation between gear wheel and thrust washer changes direction.

Figure 7 illustrates the contact pressure of all contact elements introduced in the model when the differential is in the drive to rear mode. The time evolution of contact pressure is depicted in Fig. 8 where it can be observed that at each time and for each working mode, three contact elements are active and two are inactive. The contact between thrust washers \#8 et \#10 (cf. Fig. 5) is the only contact element always active. The drive modes $([0 ; 7] \mathrm{s}$ and $[16 ; 24] \mathrm{s})$ involve the contacts between the sun gear and the washer \#7 and between the internal gear and the washer \#11. On the other hand, the contacts between the coupling and the washer \#11 and between the internal gear and washer \#9 are active for the coast modes ([8;15] s and [25;32] s). The friction is taken into account in the five contacts. Fig. 10 shows the spatial distribution of power dissipated by friction. The analysis of stresses in the thrust washers can be also provided by the simulation (Fig. 9). All these numerical data are useful to design the TORSEN differential. For instance, the internal and external radius of thrust washers or the friction coefficient of the contact can be adapted according to the locking effect wanted for each working mode. The washer thickness could also be modified thanks to the stress analysis. Further, the inclusion of this differential model in a full vehicle where the car body, suspensions, driveshafts or tyres are 


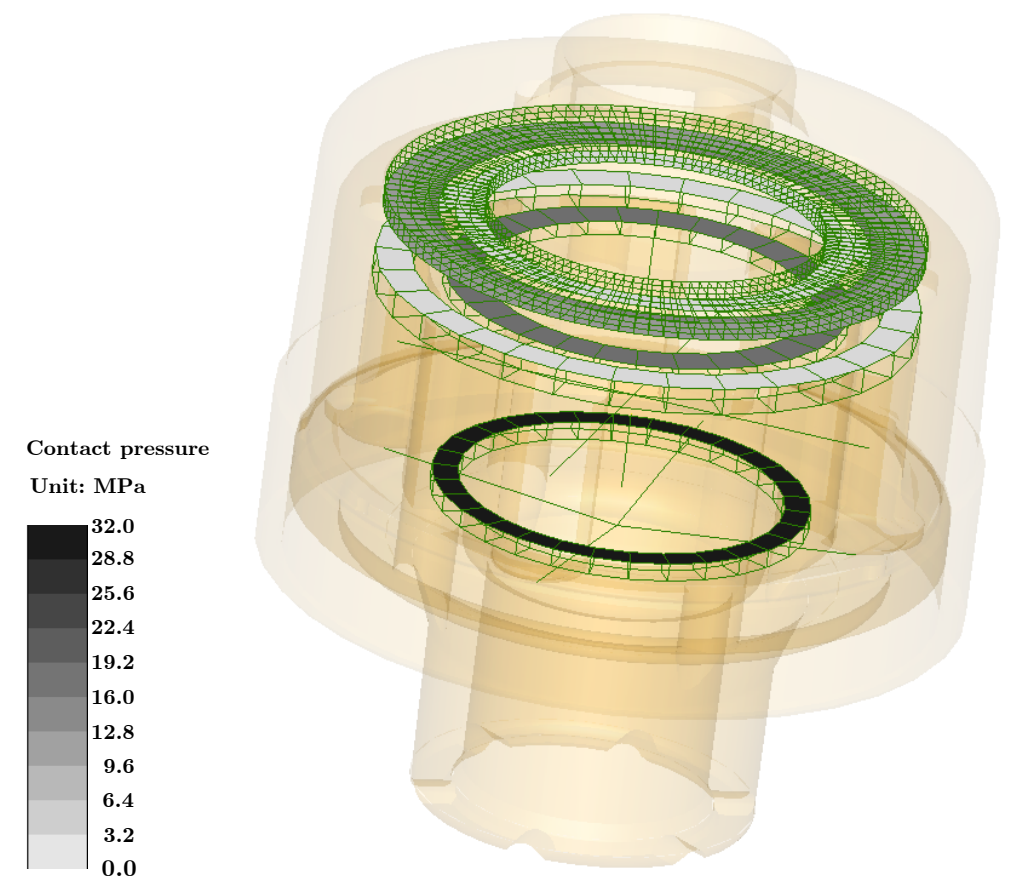

Fig. 7 Contact pressure on thrust washers ( $t=3.5 \mathrm{~s}:$ Drive to rear mode $)$

represented, would allow to study the interaction between the differential and the vehicle dynamics.

A particular attention must be put on the meshing of the thrust washers. The kind of finite element used can influence the convergence properties and the results accuracy. It could be noticed that considering the thrust washers with a volume behaviour is better than with shell finite elements. Furthermore, it is better if the contact surface is composed of quadratic elements which are thereafter extruded to obtain hexahedron elements. In order to avoid tetrahedric elements in the mesh, the geometry of the thrust washers has been slightly simplified to obtain a perfect ring shape.

The main disadvantage of this rigid/flexible contact element is the high computational time needed owing to the important number of configuration parameters required by this contact formulation. Indeed, this contact condition requires to model at least one of two contacting bodies with finite elements. For instance, in case of volume elements, the number of generalized coordinates is more than three times the number of nodes plus the Lagrange multipliers linked to the contact element attached to each slave node. For some applications, it is not always necessary to account for the flexibility of the bodies in contact. In this case, this contact element increases the size and unnecessarily complicates the model. 


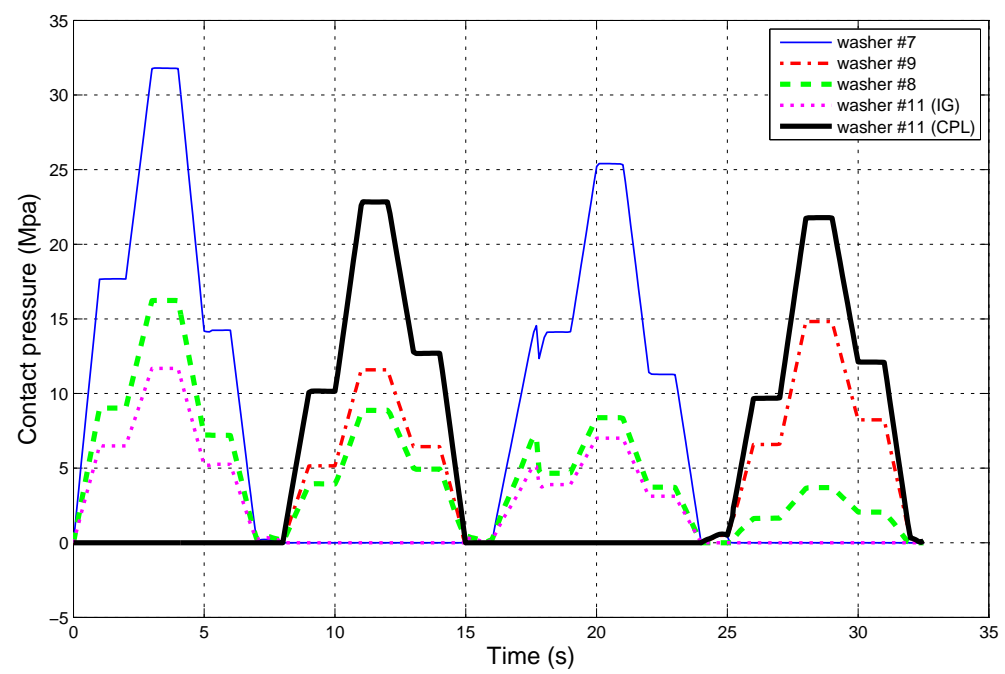

Fig. 8 Time evolution of the contact pressure in thrust washers

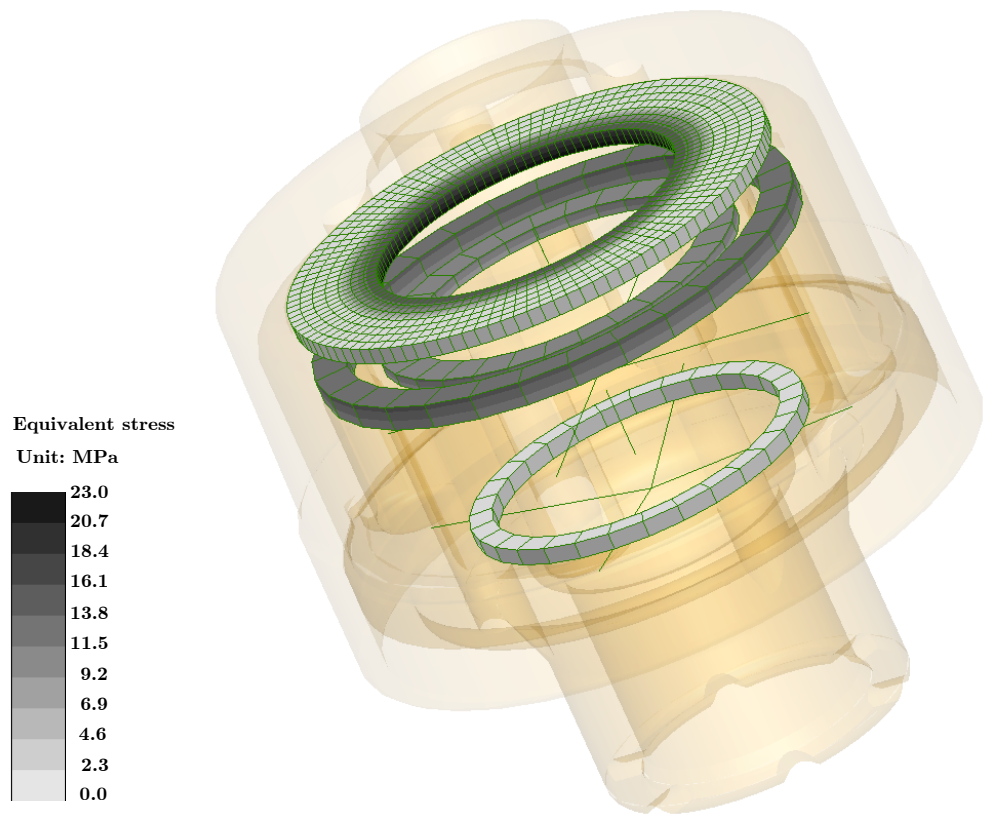

Fig. 9 Stress in the thrust washers ( $t=11.6 \mathrm{~s}:$ Coast to front mode) 

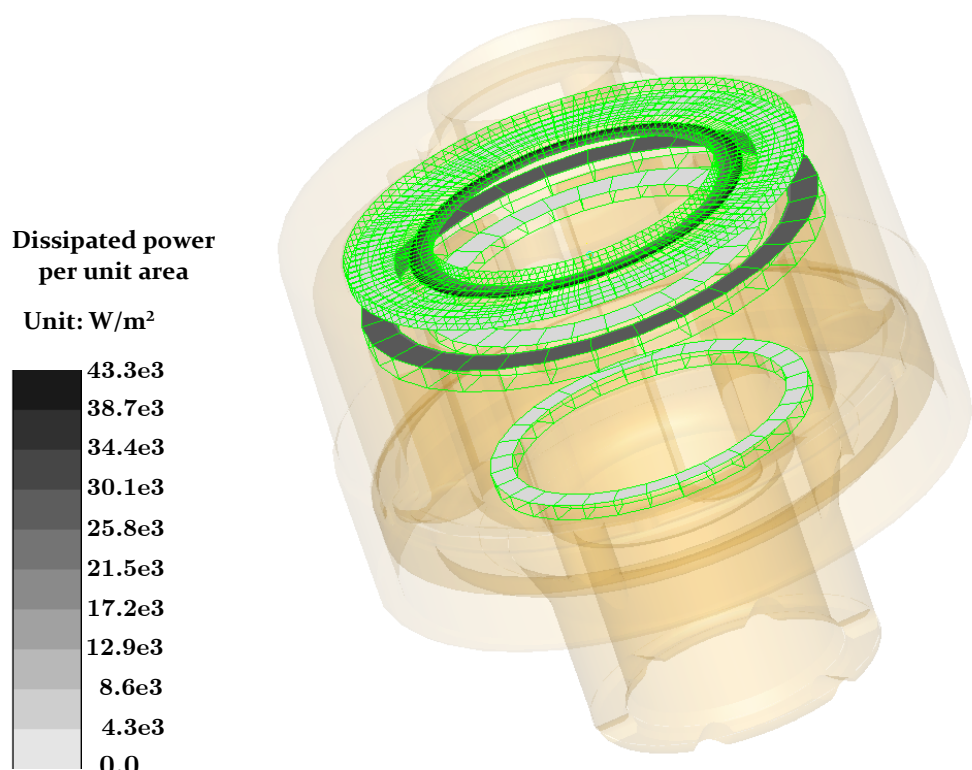

Fig. 10 Power dissipated by friction between gear wheels and thrust washers $(t=28.2 \mathrm{~s}$ : Coast to rear mode)

\subsection{Rigid-Rigid Contact}

The frictional contact formulation based on the continuous impact theory, described in Sect. 4 of this chapter, has been implemented in the user element framework of SAMCEF/MECANO. In order to test this new contact element and compare its performance with the coupled iterations method for flexible-rigid contact, the TORSEN differential has also been considered as the application system. The same kind of loading and limit conditions have been used whereas the five contact conditions have been replaced by the new contact elements.

Figure 11 shows the time evolution of the torque on the sun gear and coupling when the drive to rear mode of the differential is active (first quarter of the simulation depicted in Fig. 6). These torques can be seen as the reaction torque on the differential output shafts linked to the sun gear and the coupling because their rotation speed is prescribed whereas a torque is applied on the differential input (housing).

The spikes on curves of Fig. 11 represent the shocks due to impacts when the gear wheels move quickly at the switching time between to working modes. With the rigid/flexible contact formulation, the magnitude of these spikes is almost vanished due to the anticipating damping used to enable the algorithm convergence. The effects of impacts are are also depicted in Fig. 12 which illustrates the axial displacement of the sun gear. The rebonds of gear wheels against the various thrust washers occur at each change of differential working mode. The magnitude and the 
frequency of these rebonds depends on the restitution coefficient and the contact stiffness used for each contact element.

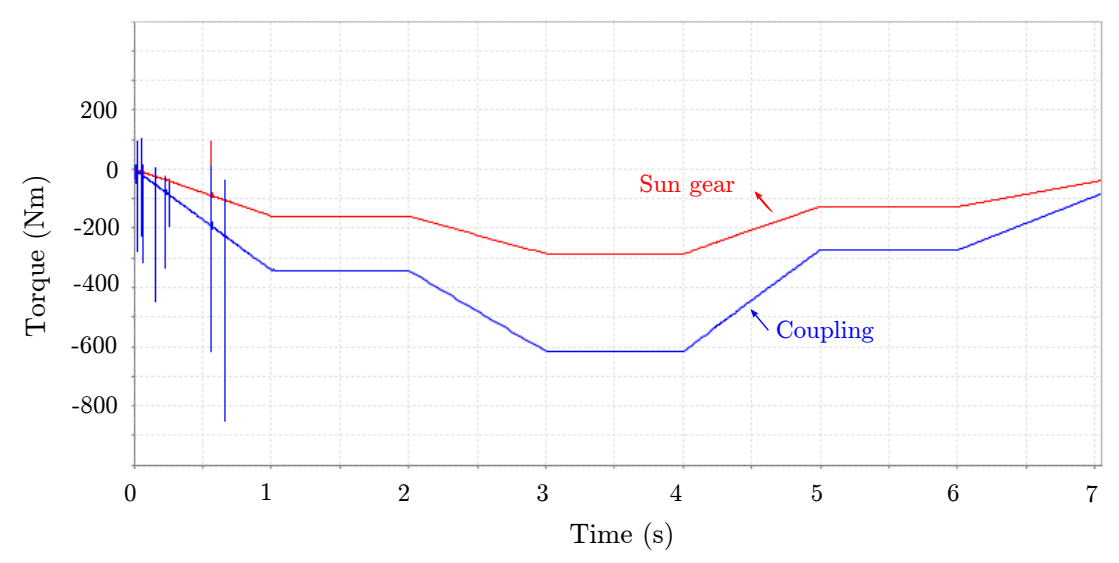

Fig. 11 Reaction torque on the sun gear and the coupling

This rigid/rigid contact condition based on the continuous impact modelling is suitable for global models of complex automotive components due to its robustness and implementation simplicity. Besides the modelling of TORSEN differential, this contact formulation could be easily extended to model the contact between plates in clutch or between synchronization devices in gear boxes.

Compared with the previous contact element, the main advantage is the reduction of the computation time by a factor of five. Moreover, the transient behaviour before and after the impact is better represented because it is not mandatory to add an anticipating damping before the impact. The amount of numerical damping needed to enable the convergence is less than for the previous contact formulation. A Hilbert-Hughes-Taylor integration scheme can be used whereas the ChungHulbert method with the maximum of numerical damping allowed was necessary for the rigid/flexible contact. The contact stiffness and the restitution coefficient are the only two parameters needed for this contact element. Their value have to be determined according to several criteria: material properties of the two contacting bodies, geometry of the contact surfaces,... Most of the time, in order to find accurate and reliable values for theses parameters, some physical experiments have to be carried out. For intricate configurations, the experimental measures often require a complicated set-up and expensive instruments. Another way to fix the restitution coefficient and contact stiffness addresses the achievement of detailed numerical simulations. These later are carried out on a faster time scale than the multibody model simulation within the contact condition is included. Nevertheless, in both case these operations are time consuming and request a lot of resources. This is one drawback of this contact formulation based on the restitution coefficient. Currently, neither experiments nor detailed numerical simulations have been carried out for 

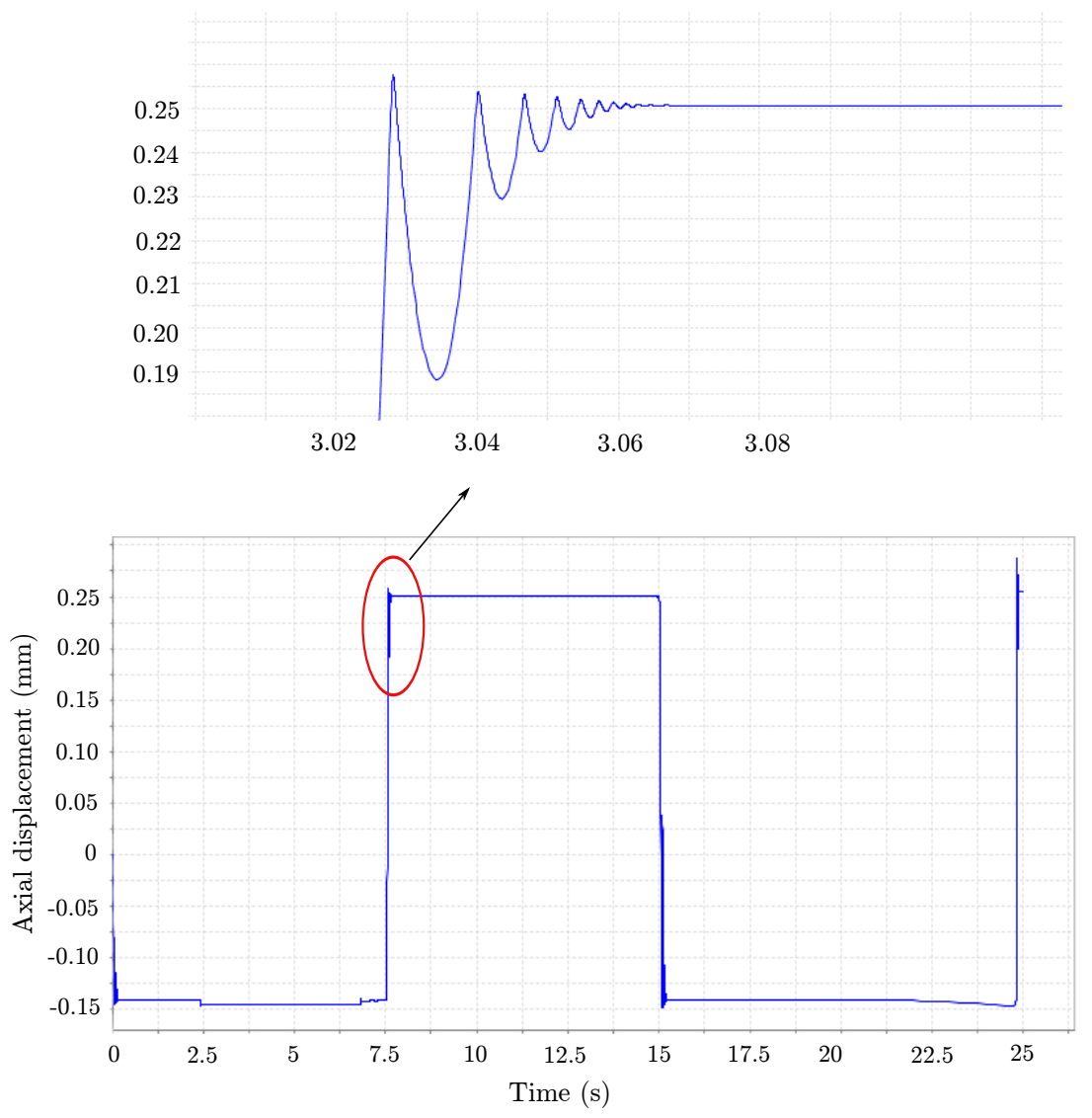

Fig. 12 Axial displacement of the sun gear $(e=0.8)$

the TORSEN differential model. For the five contact elements, the contact stiffness has been chosen according to the Young's modulus of the steal used for the gear wheels and thrust washers. The restitution coefficient has been prescribed to a value commonly used for contact between two metallic bodies $(e=0.8)$.

\section{Conclusions}

This chapter is about the modelling of unilateral contacts included in automotive transmission components. These contacts are essential for the correct operation of mechanical devices such as gear boxes, differentials or clutches. Physical phenomena like impacts or stick-slip highly influence the dynamic behaviour of the full 
automotive driveline but they are particularly difficult to model accurately and efficiently.

Two different contact formulations have been considered in this work. Both contact elements have been used to represent the contacts between gear wheels and thrust washers in a full TORSEN differential model. The friction has been taken into account in all contact elements because friction torques are fundamental for the locking effect of this kind of limited slip differential.

The first one uses the coupled iterations method and is able to model contact between two flexible bodies modelled with finite elements or between a rigid body and a flexible body. During the first step of this contact algorithm, each slave node on the contact surface of the first body is projected on the master face of the second body submitted to the contact condition. From this projection, an associated distance sensor in the normal and tangential directions is created. The relative displacement in theses directions allows to determine the contact status: inactive or active contact, sticking or sliding contact. The second step addresses the definition of the contact condition according to the contact status. An augmented Lagrangian approach is used to express the three kinematic constraints. However if impacts occur, some convergence problems can appear. For the simulation of the TORSEN differential, the augmented Lagrangian approach has to be replaced by a full penalty method in order to have a robust model. A dynamic simulation including the four working modes has been performed and has been globally validated by comparison with experimental data [22].

The second contact formulation implemented is defined between two rigid bodies and is based on the continuous impact theory. With this method, a restitution coefficient is used to account for the kinetic energy loss during the impact process. The contact force is determined by an impact law which depends on the contact stiffness, the restitution coefficient and the relative local penetration and penetration velocity between the two rigid bodies. This second formulation is more robust to represent the transient behavior close to the impact and enables to greatly reduce the number of degrees of freedom as well as the computing time. However, in contrast to the first contact formulation (flexible/flexible or rigid/flexible), the analysis of deformations and stresses in the contacting bodies is not available with this global contact model.

For global applications like full automotive drivetrain systems, the two contact formulations presented in this chapter could be used together inside the same model. For instance, the coupled iterations method between flexible bodies could be used for the contacts included within the transmissions devices accurately modelled. While for the driveline components coarsely modelled, the rigid-rigid contact formulation could be used to represent these contact conditions in a more global way. Further, the two contact models could be extended in order to capture specific phenomena such as stick-slip often encountered in mechanical transmission devices. 


\section{Acknowledgements}

The author, Geoffrey Virlez would like to acknowledge the Belgian National Fund for Scientific research (FRIA) for its financial support.

\section{References}

1. Acary, V., Brogliato, B.: Numerical methods for nonsmooth dynamical systems. Lecture Notes in Applied and Computational Mechanics 35, 540 (2008)

2. Arnold, M., Brüls, O.: Convergence of the generalized- $\alpha$ scheme for constrained mechanical systems. Multibody System Dynamics 18(2), 185-202 (2007)

3. Blundell, M., Harty, D.: The Multibody Systems Approach to Vehicle Dynamics. Elsevier Butterworth-Heinemann Publications (2004)

4. Cardona, A.: Flexible three dimensional gear modelling. European journal of computational mechanics 4(5-6), 663-691 (1995)

5. Christensen, P., Klarbring, A., Pang, J., Strömberg, N.: Formulation and comparison of algorithms for frictional contact problems. International Journal for Numerical Methods in Engineering. 42, 145-173 (1998)

6. Chung, J., Hulbert, G.: A time integration algorithm for structural dynamics with improved numerical dissipation: The generalized- $\alpha$ method. ASME Journal of Applied Mechanics 60, 371-375 (1993)

7. Drab, C., Engl, H., Haslinger, J., Offner, G.: Dynamic simulation of crankshaft multibody systems. Mulibody Syst Dyn 22, 133-144 (2009)

8. Fichera, G., Lacagnina, M., Petrone, F.: Modelling of torsion beam rear suspension by using multibody method. Mulibody System Dynamics 12, 303-313 (2004)

9. Géradin, M., Cardona, A.: Flexible multibody dynamics. John Wiley \& Sons (2001)

10. Gonalves, J.P., Ambrosio, J.A.C.: Optimization of vehicle suspension systems for improved comfort of road vehicles using flexible multibody dynamics. Nonlinear Dynamics 34, 113131 (2003)

11. Hartmann, S., Ramm, E.: A mortar based contact formulation for non-linear dynamics using dual lagrange multipliers. Finite Elements in Analysis and Design 44, 245-258 (2008)

12. Hjiaj, M., Feng, Z.Q., De la Saxcé, G., Mróz, Z.: Three-dimensional finite element computations for frictional contact problems with non-associated sliding rule. International Journal for Numerical Methods in Engineering 60, 2045-2076 (2004)

13. Jean, M.: The non-smooth contact dynamics method. Comput. Methods Appl. Engrg 177, 235-257 (1999)

14. Kogut, L., Etsion, I.: Elastic-plastic contact analysis of a sphere and a rigid flat. Journal of Applied Mechanics 69, 657-662 (2002)

15. Lankarani, H., Nikravesh, P.: Continuous contact force models for impact analysis in multibody analysis. Nonlinear Dynamics 5, 193-207 (1994)

16. Lens, E., Cardona, A.: A nonlinear beam element formulation in the framework of an energy preserving time integration scheme for constrained multibody systems dynamics. Computers and Structures 86, 47-63 (2008)

17. Ma, Z.D., Perkins, N.C.: An efficient multibody dynamics model for internal combustion engine systems. Multibody Systems Dynamics 10, 363-391 (2003)

18. Potenza, R., F, D.J., Richardson: A model for simulating the instantaneous crank kinematics and total mechanical losses in a multicylinder in-line engine. International Journal of Engine Research 8(4), 379-397 (2007)

19. Puso, M.: A 3d mortar method for solid mechanics. International Journal for Numerical Methods in Engineering 69, 657-662 (2004) 
20. Seifried, R., Schiehlen, W., Eberhard, P.: The role of the coefficient of restitution on impact problems in multi-body dynamics. Proc. IMechE, Part K:J. Multi-body Dynamics 224, 279$306(2010)$

21. Stronge, W.J.: Impact mechanics. Cambridge University Press, Cambridge (2000)

22. Virlez, G., Brüls, O., Poulet, N., Duysinx, P.: Simulation of differentials in four-wheel drive vehicles using multibody dynamics. In: Proceedings of the ASME 2011 International Design Engineering Technical Conferences Computers and Information in Engineering Conference IDETC/CIE 2011, August 29-31, 2011, Washington, DC, USA

23. Yang, B., Laursen, T.A.: A mortar-finite element approach to lubricated contact problems. Comput. Methods Appl. Mech. Engrg. 198, 3656-3669 (2009) 